\title{
DIFFERENTIAL GEOMETRY OF MATRIX INVERSION
}

\author{
MARKO HUHTANEN*
}

\begin{abstract}
Essentially, there exists just the dimension segregating (square) matrix subspaces. In view of algebraic operations, this quantity is not particularly descriptive. For differential geometric information on matrix inversion, the second fundamental form is found for the set of inverses of the invertible elements of a matrix subspace. Several conditions for this form to vanish are given, such as being equivalent to a Jordan subalgebra. Global measures of curvature are introduced in terms of an analogy of the Nash fiber.
\end{abstract}

\section{Introduction}

The inverse of a nonsingular matrix is undoubtedly the most sought object in linear algebra. ${ }^{1}$ There exists an analogous, albeit far more geometrical notion for the Grassmannian manifolds of square matrices [12], [13]. To this end, let $\mathscr{V}$ be a matrix subspace of $\mathrm{C}^{n \times n}$ over C (or R) possessing invertible elements. Such subspaces are called nonsingular. Otherwise, $\mathscr{V}$ is called singular if it does not contain invertible elements. (For singular matrix subspaces, see [5] and references therein.) For a nonsingular matrix subspace $\mathscr{V}$, set

$$
\operatorname{Inv}(\mathscr{V})=\left\{V^{-1}: V \in \mathscr{V} \cap \operatorname{GL}(n, \mathrm{C})\right\},
$$

where $\operatorname{GL}(n, \mathrm{C})$ denotes the group of invertible $n$-by- $n$ complex entried matrices. Since giving a simple characterization of $\operatorname{Inv}(\mathscr{V})$ is not easy in general, in this paper we are concerned with the differential geometric aspects of the problem.

In connection with discretizing partial differential equations, the problem of characterizing the inverses received attention already in the late 1950s [2], [3]. (See [24] for related historical remarks.) More recently, understanding the structure of $\operatorname{Inv}(\mathscr{V})$ has turned out to be central in matrix factorization problems and large scale numerical linear algebra of preconditioning [13], [4]. It is noteworthy that in preconditioning $n$ is very large whereas $\operatorname{dim} \mathscr{V} \ll n^{2}$,

\footnotetext{
* Supported by the Academy of Finland.

Received 27 April 2009, in revised form 9 October 2009.

${ }^{1}$ The inverse is actually rarely computed. An appropriate factorization of the matrix suffices since, in practice, only operations with the inverse are needed.
} 
typically $\operatorname{dim} \mathscr{V}=O(n)$. In the generalized eigenvalue problem the dimension of the matrix subspace is just two. In applications $\operatorname{Inv}(\mathscr{V})$ is hence often dimensionwise closer to a curve than to a hypersurface.

To measure how curved $\operatorname{Inv}(\mathscr{V})$ is locally, a Riemannian geometric approach yields us the second fundamental form II of $\operatorname{Inv}(\mathscr{V})$. (How to interpret II geometrically, see [16, Chapter 8].) The sectional curvatures of $\operatorname{Inv}(\mathscr{V})$ can then be found with II. The second fundamental form is shown to vanish identically if and only if $\mathscr{V}$ is a so-called invertible matrix subspace. Several equivalent conditions for this to happen are given. Most notably, $\mathscr{V}$ is invertible if and only if $\mathscr{V}$ is equivalent to a Jordan subalgebra. Hence the concept is linked with a classical notion of nonassociative algebras, resembling the way how the exponential function is linked with Lie subalgebras. Related with linear estimation theory, this same link exists with statistics in case of real symmetric matrix subspaces [23].

For a global measure of curvature, we introduce an analogue of the Nash fiber from algebraic geometry by forming the set of limits of tangent spaces at the zero matrix. This is somewhat nonstandard since the zero matrix is merely a boundary point of $\operatorname{Inv}(\mathscr{V})$. However, homogeneity of $\operatorname{Inv}(\mathscr{V})$ makes this natural. Then the grade of $\mathscr{V}$ is defined as the span of the tangent spaces of $\operatorname{Inv}(\mathscr{V})$. The grade of $\mathscr{V}$ is compared with the dimension of $\mathscr{V}$.

The paper is organized as follows. Section 2 is concerned with the properties of the Grassmannian $\mathrm{Gr}_{k}\left(\mathrm{C}^{n \times n}\right)$. From the view-point of linear algebra, its elements can be regarded as being either nonsingular or singular. To distinguish elements of $\mathrm{Gr}_{k}\left(\mathrm{C}^{n \times n}\right)$ qualitatively, in Section 3 nonsingular matrix subspaces are treated as Riemannian manifolds. The curvature of $\operatorname{Inv}(\mathscr{V})$ is inspected. In Section 4 global measures of curvature are considered.

\section{The Grassmannian $\mathrm{Gr}_{k}\left(\mathrm{C}^{n \times n}\right)$}

In what follows, we are concerned with the Grassmannian $\mathrm{Gr}_{k}\left(\mathrm{C}^{n \times n}\right)$ consisting of $k$ dimensional subspaces of $\mathrm{C}^{n \times n}$ over $\mathrm{C}^{2}$ One can proceed completely analogously with the Grassmannian of $k$ dimensional matrix subspaces of $\mathrm{C}^{n \times n}$ over R. This relaxed structure is of equal importance including, for example, subspaces of Hermitian matrices.

On $\mathrm{Gr}_{k}\left(\mathrm{C}^{n \times n}\right)$ there exists a standard Riemannian structure giving rise to the respective distance function. For a review on distance functions on a Grassmannian, see [7, p. 337]. Since the geometry of $\mathrm{Gr}_{k}\left(\mathrm{C}^{n \times n}\right)$ is not our main

\footnotetext{
${ }^{2}$ As opposed to matrix groups and matrix subalgebras, matrix subspaces have not received much attention. In operator theory, a closed subspace of $B(H)$, the algebra of all bounded operators on a Hilbert space $H$, is called an operator space. Associated operator space theory has developed rapidly in recent years [8], [20].
} 
object of interest, we employ a metric that can be defined more readily. To this end, on any matrix subspace $\mathscr{V} \in \mathrm{Gr}_{k}\left(\mathrm{C}^{n \times n}\right)$ we use the standard inner product

$$
\left(V_{1}, V_{2}\right)=\operatorname{tr}\left(V_{2}^{*} V_{1}\right)
$$

with $V_{1}, V_{2} \in \mathscr{V}$. Equipped with this, suppose $\mathscr{V}, \mathscr{W} \in \mathrm{Gr}_{k}\left(\mathrm{C}^{n \times n}\right)$ and denote by $\mathbf{P}_{\mathscr{V}}$ and $\mathbf{P}_{\mathscr{W}}$ the orthogonal projectors on $\mathrm{C}^{n \times n}$ onto $\mathscr{V}$ and $\mathscr{W}$. Then

$$
d(\mathscr{V}, \mathscr{W})=\left\|\mathbf{P}_{\mathscr{V}}-\mathbf{P}_{\mathscr{W}}\right\|
$$

yields a metric on $\mathrm{Gr}_{k}\left(\mathrm{C}^{n \times n}\right)$, where $\|\cdot\|$ denotes the operator norm. By $\|\cdot\|_{F}$ we denote the Frobenius norm.

Usually the points of a Grassmannian do not possess any particular distinctive qualitative properties. With $\mathrm{Gr}_{k}\left(\mathrm{C}^{n \times n}\right)$ this is not so.

Definition 2.1. A matrix subspace $\mathscr{V} \in \mathrm{Gr}_{k}\left(\mathrm{C}^{n \times n}\right)$ is nonsingular (singular) if the determinant function does not (does) vanish identically on $\mathscr{V}$.

With $k=1$ we are dealing with the familiar notion of nonsingularity of a matrix. If $\mathscr{V}$ is nonsingular, then its subset consisting of invertible $V \in \mathscr{V}$ is open and dense [12].

Regarding the terminology, nonsingularity is generic as follows.

THEOREM 2.2. The set of nonsingular elements of $\mathrm{Gr}_{k}\left(\mathrm{C}^{n \times n}\right)$ is open and dense.

Proof. For openness, if $\mathscr{V}$ is nonsingular, then the determinant function, by its continuity, cannot vanish identically on matrix subspaces sufficiently close to $\mathscr{V}$.

For denseness, suppose $\mathscr{V}$ is singular. (This forces $k<n^{2}$.) Take any $V \in \mathscr{V}$ of unit Frobenius norm. There exists an arbitrarily small $\lambda \in \mathrm{C}$ in modulus such that $\lambda I-V$ is invertible. Orthogonalize such a matrix $\lambda I-V$ against $\mathscr{V}$ to have $\lambda I-V=\hat{V}+E$ with $\hat{V} \in \mathscr{V}$ and $E$ in the orthogonal complement of $\mathscr{V}$. Clearly, $\|E\|_{F} \leq\|\lambda I\|_{F}$. Form an orthonormal basis $V_{1}, \ldots, V_{k}$ of $\mathscr{V}$ by setting $V_{1}=\hat{V} /\|\hat{V}\|_{F}$. Denote by $\mathscr{W}$ the span of $V_{1}+E /\|\hat{V}\|_{F}, V_{2}, \ldots, V_{k}$. Then $\mathscr{W}$ is nonsingular and of the same dimension as $\mathscr{V}$. Moreover, $d(\mathscr{V}, \mathscr{W})$ equals the norm of the linear operator

$$
V_{1}\left(\cdot, V_{1}\right)-\frac{1}{1+\left(\|E\|_{F} /\|\hat{V}\|_{F}\right)^{2}}\left(V_{1}+E /\|\hat{V}\|_{F}\right)\left(\cdot, V_{1}+E /\|\hat{V}\|_{F}\right)
$$

on $C^{n \times n}$. As $\lambda$ approaches zero, $E$ approaches zero and $\|\hat{V}\|_{F}$ approaches one. Thus, there is an invertible matrix subspace arbitrarily close to $\mathscr{V}$. 
We do not know what is the largest $k$ for which there are necessarily singular elements in $\mathrm{Gr}_{k}\left(\mathrm{C}^{n \times n}\right)$. It is of order $k=O\left(n^{2}\right)$ though. (For $k=n(n-1)$, simply take a matrix subspace with a zero row or column.) Typically matrix subspaces appearing in factorization problems satisfy $k=O(n)$ [12].

With matrices, mere nonsingularity is not at all sufficient for reliably performing inversion; in practice the condition number is used to assess nonsingularity. For an analogy, for $1 \leq j \leq n$, denote by $\sigma_{j}(V)$ the singular values of a matrix $V \in \mathrm{C}^{n \times n}$. For a nonsingular matrix subspace $\mathscr{V}$, even though not readily computable, set

$$
\kappa(\mathscr{V})=\min _{V \in \mathscr{V} \cap \mathrm{GL}(n, \mathrm{C})} \frac{\sigma_{1}(V)}{\sigma_{n}(V)}
$$

to measure its ill-conditioning. In case $\operatorname{dim} \mathscr{V}=1$ we are dealing with the standard condition number of any matrix $V$ spanning $\mathscr{V}$. Clearly, $\kappa(\mathscr{V}) \geq 1$ with the equality holding if and only if $\mathscr{V}$ contains a unitary matrix.

The group $\mathrm{GL}(n, \mathrm{C}) \oplus \mathrm{GL}(n, \mathrm{C})$ acts on $\mathrm{Gr}_{k}\left(\mathrm{C}^{n \times n}\right)$ according to

$$
(X \oplus Y) \mathscr{V}=X \mathscr{V} Y^{-1} \text {. }
$$

This is a natural action preserving, e.g., nonsingularity. The orbit of $\mathscr{V}$ is then a subset of $\mathrm{Gr}_{k}\left(\mathrm{C}^{n \times n}\right)$ defined as

$$
O(\mathscr{V})=\left\{X \mathscr{V} Y^{-1}: X \oplus Y \in \operatorname{GL}(n, \mathrm{C}) \oplus \operatorname{GL}(n, \mathrm{C})\right\} .
$$

Definition 2.3. Matrix subspaces $\mathscr{V}$ and $\mathscr{W}$ are said to be equivalent if they are on the same orbit, i.e., there exist invertible matrices $X, Y \in \mathrm{C}^{n \times n}$ such that $\mathscr{W}=X \mathscr{V} Y^{-1}$.

If $X$ and $Y$ can be chosen unitary, then $\mathscr{V}$ and $\mathscr{W}$ are said to be unitarily equivalent. If $X=Y$, then $\mathscr{V}$ and $\mathscr{W}$ are said to be similar.

Matrix subspaces on the same orbit are in many ways indistinguishable. Hence there are good reasons to look for elements of $O(\mathscr{V})$ with simple properties. With $k=2$ this is always possible as there is an element whose members commute.

ExAMPLE 2.4. In applications, the Grassmannian $\mathrm{Gr}_{2}\left(\mathrm{C}^{n \times n}\right)$ appears in connection with the generalized eigenvalue problem

$$
V_{1} x=\lambda V_{2} x
$$

with $x \neq 0$. Namely, assume $\mathscr{V}=\operatorname{span}\left\{V_{1}, V_{2}\right\} \in \mathrm{Gr}_{2}\left(\mathrm{C}^{n \times n}\right)$ is nonsingular. ${ }^{3}$ Then $\mathscr{V}$ is equivalent to a matrix subspace $\mathscr{W}$ with commuting elements. For

\footnotetext{
${ }^{3}$ If $\mathscr{V}$ is singular, then the respective generalized eigenvalue problem (3) is sometimes called ill-disposed. For the generalized eigenvalue problem, see [9], [19].
} 
this, take $X=I$ and $Y$ to be an invertible element of $\mathscr{V}$. (By this trick, $\mathscr{V} Y^{-1}$ also contains the scalars.) This transformation is of enormous importance for solving the generalized eigenvalue problem in practice.

Because of this, $\mathrm{Gr}_{k}\left(\mathrm{C}^{n \times n}\right)$ for $k>2$ can be regarded as having a much more "noncommutative" structure than $\mathrm{Gr}_{2}\left(\mathrm{C}^{n \times n}\right)$. Let us emphasize this profound difference in terms of the following proposition.

Proposition 2.5. Assume $n \geq 2$ and $k>2$. Then there exists $\mathscr{V} \in$ $\mathrm{Gr}_{k}\left(\mathrm{C}^{n \times n}\right)$ whose orbit does not contain a matrix subspace with commuting elements.

Proof. To recover whether a nonsingular $\mathscr{V}=\operatorname{span}\left\{V_{1}, V_{2}, \ldots, V_{k}\right\}$ is equivalent to a matrix subspace $\mathscr{W}$ with commuting elements, it is necessary and sufficient that $X V_{j} Y^{-1}$ and $X V_{l} Y^{-1}$ commute with some invertible $X, Y \in$ $\mathrm{C}^{n \times n}$, for $1 \leq j<l \leq k$. Hence consider the problem of finding such matrices $X$ and $Y$.

Since $\mathscr{V}$ is nonsingular, its subset consisting of invertible elements is open and dense. Thereby we may assume that $V_{j}$ are all invertible, possibly after perturbing them slightly in $\mathscr{V}$. For the commutativity, consider the linear maps

$$
M \longmapsto V_{l} V_{j}^{-1} M-M V_{j}^{-1} V_{l}
$$

on $C^{n \times n}$, for $1 \leq j<l \leq k$. A matrix subspace $\mathscr{W}$ with commuting elements exists if and only if the intersection of the nullspaces of (4) contains an invertible element $M$. Then the condition $M=Y^{-1} X$ determines $X$ and $Y$ for an equivalence.

With this, it suffices to consider the case $k=3$ and $n=2$. Take

$$
V_{1}=I, \quad V_{2}=\left[\begin{array}{ll}
1 & 1 \\
0 & 1
\end{array}\right] \quad \text { and } \quad V_{3}=\left[\begin{array}{ll}
1 & 0 \\
1 & 1
\end{array}\right]
$$

for which the intersection of the nullspaces of (4) is just the zero matrix.

A matrix subspace is said to be symmetric if all its elements are symmetric matrices. Symmetric matrix subspaces appear in the study of determinantal hypersurfaces [21] and in statistics [23].

Proposition 2.6. A nonsingular $\mathscr{V} \in \mathrm{Gr}_{2}\left(\mathrm{C}^{n \times n}\right)$ is equivalent to a symmetric matrix subspace.

Proof. Since $\mathscr{V}$ is nonsingular, it is equivalent to a matrix subspace of the form $\operatorname{span}\{I, W\}$ with $W \in \mathrm{C}^{n \times n}$. Since any matrix is the product of two symmetric matrices, we have $W=S_{1} S_{2}$ for two symmetric matrices with at least $S_{2}$ invertible. Hence $\mathscr{V}$ is equivalent to $\operatorname{span}\left\{S_{2}^{-1}, S_{1}\right\}$. 
For a nonsingular matrix subspace $\mathscr{V}$, there are always elements of $O(\mathscr{V})$ containing the scalars. Such elements are somewhat easier to analyze and thereby of interest. For example, in operator space theory, a matrix subspace $\mathscr{V}$ over $\mathrm{C}$ is said to be an operator system if it contains the scalars and is closed under the Hermitian transposition, i.e., if $V \in \mathscr{V}$, then $V^{*} \in \mathscr{V}$ [8].

EXAMPLE 2.7. The set of Toeplitz and Hankel matrices are unitarily equivalent matrix subspaces often encountered in practice. ${ }^{4}$ For the unitary equivalence, take $X$ to be the permutation with ones on the antidiagonal and $Y=I$. The set of Toeplitz matrices contains the scalars.

\section{The set $\operatorname{Inv}(\mathscr{V})$ as a Riemannian submanifold}

Next, in view of matrix inversion, we consider ways to qualitatively distinguish nonsingular elements of $\mathrm{Gr}_{k}\left(\mathrm{C}^{n \times n}\right)$. To this end, for a nonsingular matrix subspace $\mathscr{V}$ over C (or R) denote the set of its inverses by

$$
\operatorname{Inv}(\mathscr{V})=\left\{V^{-1}: V \in \mathscr{V} \cap \operatorname{GL}(n, \mathrm{C})\right\} .
$$

As a manifold this is covered by a single chart which can be obtained after fixing a basis of $\mathscr{V}$ and performing the inversion. In particular, equipped with the standard inner product $(1), \operatorname{Inv}(\mathscr{V})$ can be regarded as a smooth Riemannian submanifold of $\mathrm{C}^{n \times n}$ of dimension $k$. Then $\mathrm{C}^{n \times n}$ is equipped with the Frobenius norm $\|\cdot\|_{F}$.

Plainly, $\operatorname{Inv}(\mathscr{V})$ is a homogeneous set, i.e., we have

$$
t \operatorname{Inv}(\mathscr{V})=\operatorname{Inv}(\mathscr{V})
$$

for any $0 \neq t \in \mathrm{C}$ (or R). (However, unlike in $\mathscr{V}$, the sum operation is not closed in $\operatorname{Inv}(\mathscr{V})$ in general.) Therefore it is occasionally natural to treat $\operatorname{Inv}(\mathscr{V})$ as a projective variety.

For the equivalence there holds

$$
\operatorname{Inv}\left(X \mathscr{V} Y^{-1}\right)=Y \operatorname{Inv}(\mathscr{V}) X^{-1} .
$$

Similarly, for the transposition we have $\operatorname{Inv}\left(\mathscr{V}^{T}\right)=\operatorname{Inv}(\mathscr{V})^{T}$.

Many quantities of interest can be computed with the help of Cramer's rule for $\operatorname{Inv}(\mathscr{V})$. (Consider, for instance, finding lengths of curves.) Because of its high computational complexity, Cramer's rule is really available in small dimensions only. Therefore its usage is avoided in what follows.

After these preliminary remarks, let us construct the tangent vectors to see that the tangent bundle of $\operatorname{Inv}(\mathscr{V})$ admits a useful extrinsic characterization.

\footnotetext{
${ }^{4}$ A Toeplitz (Hankel) matrix has constant diagonals (antidiagonals).
} 
For this, take a smooth curve with the Taylor series $c(t)=W t+U t^{2}+\cdots$ with the coefficients belonging to $\mathscr{V}$. Let $V^{-1} \in \operatorname{Inv}(\mathscr{V})$. Then the Neumann series expansion yields for

(7)

$$
\begin{aligned}
(V+c(t))^{-1} & =\left(\sum_{j=0}^{\infty}(-1)^{j}\left(V^{-1} c(t)\right)^{j}\right) V^{-1} \\
& =V^{-1}-V^{-1} W V^{-1} t+V^{-1}\left(W V^{-1} W-U\right) V^{-1} t^{2}+\cdots
\end{aligned}
$$

for small enough $|t|$. Collecting the linear terms of such curves, we can conclude that at $V^{-1} \in \operatorname{Inv}(\mathscr{V})$ the tangent space is

$$
V^{-1} \mathscr{V} V^{-1} \text {. }
$$

In practice, tangent spaces appear in numerical analysis and perturbation theory.

Example 3.1. Suppose the inverse of $V$ is available. Then the classical perturbation theory yields

$$
(V+W)^{-1} \approx V^{-1}-V^{-1} W V^{-1}
$$

for the inverse of $V+W$. This approximation belongs to $V^{-1} \mathscr{V} V^{-1}$ by the fact that $V^{-1}-V^{-1} W V^{-1}=V^{-1}(V-W) V^{-1}$. This constraint is somewhat confusing since there are no particular reasons for the tangent space $V^{-1 \mathscr{V}} V^{-1}$ to contain the inverse of $V+W$.

In the following case the tangent space is guaranteed to contain the inverse.

Definition 3.2. Let $\mathscr{V}$ and $\mathscr{W}$ be two nonsingular matrix subspaces of $\mathrm{C}^{n \times n}$ over C (or R). If

$$
\operatorname{Inv}(\mathscr{V})=\mathscr{W} \cap \mathrm{GL}(n, \mathrm{C}),
$$

then we say that $\mathscr{W}$ is the inverse of $\mathscr{V}$.

If $\mathscr{V}$ has an inverse, then the tangent spaces of $\operatorname{Inv}(\mathscr{V})$ are independent of the base point $V^{-1} \in \operatorname{Inv}(\mathscr{V})$. Then the closure of $\operatorname{Inv}(\mathscr{V})$ equals $V^{-1 \mathscr{V}} V^{-1}$ [13]. Hence the inverse is unique. We denote the inverse by $\mathscr{V}^{-1}$ and say that $\mathscr{V}$ is invertible. Stated equivalently in terms of the Grassmannians, a nonsingular $\mathscr{V} \in \mathrm{Gr}_{k}\left(\mathrm{C}^{n \times n}\right)$ is invertible if the closure of $\operatorname{Inv}(\mathscr{V})$ belongs to $\mathrm{Gr}_{k}\left(\mathrm{C}^{n \times n}\right)$. This also explains the terminology used: then $\mathscr{V}$ can be regarded as being invertible in $\mathrm{Gr}_{k}\left(\mathrm{C}^{n \times n}\right)$.

By (6), the whole orbit of an invertible matrix subspace consists of invertible matrix subspaces. 
Invertible matrix subspaces can be characterized as follows.

THEOREM 3.3 ([13]). $\mathscr{V}$ is an invertible subspace of $\mathrm{C}^{n \times n}$ over $\mathrm{C}($ or $\mathrm{R})$ if and only if $\mathscr{V}=V \hat{\mathscr{V}}$ with an invertible $V \in \mathrm{C}^{n \times n}$ and a matrix subspace $\hat{\mathscr{V}}$ of $\mathrm{C}^{n \times n}$ over $\mathrm{C}($ or $\mathrm{R})$ that is polynomially closed. ${ }^{5}$

Moreover, if $\mathscr{V}$ contains the scalars, then $\mathscr{V}$ is invertible if and only if $\mathscr{V}$ is polynomially closed [13]. Besides this, we do not have a good understanding of the structure of invertible matrix subspaces as a subset of $\mathrm{Gr}_{k}\left(\mathrm{C}^{n \times n}\right)$.

Invertible matrix subspaces are of central relevance for solving a certain type of matrix factorization problems [12]. On the other hand it is a challenge to give a concrete characterization of $\operatorname{Inv}(\mathscr{V})$ [13]. Since this cannot be done in general, in what follows we are concerned with describing how curved this set is. Only the case $k \geq 2$ is of interest since $k=1$, which corresponds to the standard matrix inversion, is exceptional. In the latter case the curvature vanishes by the fact that the invertibility of a matrix $V \in \mathrm{C}^{n \times n}$ can be formulated equivalently in terms of the invertibility of the respective matrix subspace $\mathscr{V}=\operatorname{span}\{V\}$ whose inverse in the invertible case is simply $\mathscr{V}^{-1}=\operatorname{span}\left\{V^{-1}\right\}$.

For a concept of curvature at a point $V^{-1} \in \operatorname{Inv}(\mathscr{V})$, on the tangent space define a quadratic function $Q_{V^{-1}}: V^{-1} \mathscr{V} V^{-1} \rightarrow \mathrm{C}^{n \times n}$ as

$$
Q_{V^{-1}}(M)=2\left(I-\mathbf{P}_{V^{-1} \mathscr{V} V^{-1}}\right) M V M .
$$

Since $\mathscr{V}$ is a subspace and thereby homogeneous, it is enough to inspect this map at the invertible elements $V$ of unit Frobenius norm.

Although we did not require (7) to be a constant speed curve ${ }^{6}$, its Neumann series expansion can be used to relate the quadratic function (10) with geodesics as follows. (For basics of differential geometry, see [16].)

Proposition 3.4. Let $\mathscr{V}$ be a nonsingular matrix subspace of $\mathrm{C}^{n \times n}$ over $\mathrm{C}$ $($ or $\mathrm{R})$. The extrinsic curvature of the geodesic in $\operatorname{Inv}(\mathscr{V})$ passing through $V^{-1}$ with the speed vector $M=-V^{-1} W V^{-1}$ equals $\left\|Q_{V^{-1}}(M)\right\|_{F}$.

Proof. Consider the coefficient $V^{-1}\left(W V^{-1} W-U\right) V^{-1}$ of the second order term in the Neumann series expansion (7) of any curve in $\operatorname{Inv}(\mathscr{V})$ passing through $V^{-1}$ with the speed vector $M$. Twice this is the acceleration of the curve at $V^{-1}$. Since $U \in \mathscr{V}$, the element $-V^{-1} U V^{-1}$ is necessarily in the tangent space $V^{-1 \mathscr{V}} V^{-1}$. Moreover, for any $W$ there exists a unique $U$ such that the second order term is in the orthogonal complement of the tangent space.

\footnotetext{
${ }^{5} \mathrm{~A}$ matrix subspace $\mathscr{V}$ of $\mathrm{C}^{n \times n}$ is said to be polynomially closed over $\mathrm{C}$ (or R) if $p(V) \in \mathscr{V}$ for every $V \in \mathscr{V}$ and every polynomial $p$ with complex (real) coefficients. Equivalently, the holomorphic functional calculus is defined in $\mathscr{V}$.

${ }^{6}$ A curve $\gamma$ is of constant speed if $\left\|\gamma^{\prime}(t)\right\|_{F}$ is independent of $t$.
} 
This choice corresponds to applying $I-\mathbf{P}_{V^{-1} \mathscr{V} V^{-1}}$ to $2 V^{-1} W V^{-1} W V^{-1}=$ $2 V^{-1} W V^{-1} V V^{-1} W V^{-1}=2 M V M$. Then, since the tangential acceleration of the curve is zero, the geodesic in $\operatorname{Inv}(\mathscr{V})$ passing through $V^{-1}$ with the speed vector $M$ is among the curves with such a choice for $U$ [16, pp. 138-139].

Consequently, the second fundamental form II of $\operatorname{Inv}(\mathscr{V})$ can be recovered with the quadratic function $Q_{V^{-1}}$ by setting $\frac{1}{2}\left(Q_{V^{-1}}\left(M_{1}+M_{2}\right)-Q_{V^{-1}}\left(M_{1}\right)-\right.$ $\left.Q_{V^{-1}}\left(M_{2}\right)\right)$ to obtain

$$
\mathrm{II}\left(M_{1}, M_{2}\right)=\left(I-\mathbf{P}_{V^{-1} \mathscr{V} V^{-1}}\right)\left(M_{1} V M_{2}+M_{2} V M_{1}\right)
$$

at $V^{-1} \in \operatorname{Inv}(\mathscr{V})$. (For this argument, see [16, p. 138].) Then, by using the Gauss equation [16, Theorem 8.4] and [16, Proposition 8.8], also the sectional curvatures of $\operatorname{Inv}(\mathscr{V})$ can immediately be found with II. Hence, the Ricci and scalar curvatures become computable.

It is of interest to observe that the second fundamental form is in no way measuring how ill-conditioned $V$ is. ${ }^{7}$

To assess how curved $\operatorname{Inv}(\mathscr{V})$ is at $V^{-1}$, it is also natural to look at

$$
\max _{\|M\|_{F}=1}\left\|Q_{V^{-1}}(M)\right\|_{F} .
$$

Unfortunately, we do not know how to compute this quantity. In particular, to assess the curvature of $\operatorname{Inv}(\mathscr{V})$ globally, it would be very informative to know the gap between

$$
\inf _{\|V\|_{F}=1, V^{-1} \in \operatorname{Inv}(\mathscr{V})} \max _{\|M\|_{F}=1}\left\|Q_{V^{-1}}(M)\right\|_{F}
$$

and

$$
\sup _{\|V\|_{F}=1, V^{-1} \in \operatorname{Inv}(\mathscr{V})} \max _{\|M\|_{F}=1}\left\|Q_{V^{-1}}(M)\right\|_{F} .
$$

Observe that $Q_{V^{-1}}$ vanishes at $M=V^{-1} V V^{-1}=V^{-1} \in V^{-1} \mathscr{V} V^{-1}$.

Invertible matrix subspaces have identically vanishing curvature in the following sense.

THEOREM 3.5. A matrix subspace $\mathscr{V}$ of $\mathrm{C}^{n \times n}$ over $\mathrm{C}($ or $\mathrm{R})$ is invertible if and only if the quadratic function (10) is identically zero for any $V^{-1} \in \operatorname{Inv}(\mathscr{V})$.

Proof. If $\mathscr{V}$ is invertible, then $W V^{-1} W \in \mathscr{V}$ for any invertible $W \in \mathscr{V}$ by the fact that then $W^{\mathscr{V}}{ }^{-1} W=\left(\mathscr{V}^{-1}\right)^{-1}=\mathscr{V}$ holds. If $W$ is singular, then there exists an invertible element of $\mathscr{V}^{-1}$ arbitrarily close to $W$. Since $\mathscr{V}$ is closed, by continuity also $W V^{-1} W \in \mathscr{V}$ if $W \in \mathscr{V}$ is singular. Consequently,

\footnotetext{
${ }^{7}$ A matrix is said to be ill-conditioned if its condition number is large.
} 
$V^{-1} W V^{-1} W V^{-1}$ belongs to $V^{-1} \mathscr{V} V^{-1}$ and therefore (10) vanishes at $M=$ $V^{-1} W V^{-1}$ for any $W \in \mathscr{V}$.

For the converse, suppose the quadratic map (10) is identically zero. Then for any $W \in \mathscr{V}$ there exists $U \in \mathscr{V}$ such that $V^{-1} W V^{-1} W V^{-1}=V^{-1} U V^{-1}$, i.e., $W V^{-1} W=U \in \mathscr{V}$. Therefore $V^{-1}=W^{-1} U W^{-1}$ whenever $W$ is invertible. Keeping now such a $W$ fixed and letting $U$ vary in $\mathscr{V}$, we can conclude that $\operatorname{Inv}(\mathscr{V})=W^{-1} \mathscr{V} W^{-1}$. Hence $\mathscr{V}$ is invertible.

This also explains why $Q_{V^{-1}}$ vanishes at $M=V^{-1}$. Let us state it as follows.

COROLlary 3.6. Let $\hat{\mathscr{V}}$ be an invertible matrix subspace of $\mathscr{V}$ containing $V$. Then $Q_{V^{-1}}$ vanishes on $V^{-1 \hat{\mathscr{V}}} V^{-1}$.

For example, the set of circulant matrices is an invertible matrix subspace contained in the set of Toeplitz matrices.

In large scale numerical linear algebra, $\operatorname{Inv}(\mathscr{V})$ is typically approximated with its tangent space at the identity, assuming the matrix subspace $\mathscr{V}$ contains the scalars. Then the tangent space is simply $\mathscr{V}$ and hence readily available. To assess how possibly useful this linearization is, we want to inspect how curved $\operatorname{Inv}(\mathscr{V})$ is at $V=\frac{1}{\sqrt{n}} I$. This leads us to consider

$$
Q_{V^{-1}}(M)=\frac{2}{\sqrt{n}}\left(I-\mathbf{P}_{\mathscr{V}}\right) M^{2}
$$

for $M \in \mathscr{V}$.

ExAmple 3.7. In analyzing the resolvent operator of a matrix $A \in \mathrm{C}^{n \times n}$, one is concerned with $\operatorname{Inv}(\mathscr{V})$ for the matrix subspace $\mathscr{V}=\operatorname{span}\{I, A\}$. At $V=\frac{1}{\sqrt{n}} I$ we need to evaluate $\frac{2}{\sqrt{n}\|A\|_{F}^{2}}\left(I-\mathbf{P}_{\mathscr{V}}\right) A^{2}$ to find (11). (For $M=$ $t_{0} I+t_{1} A$ with $t_{0}, t_{1} \in \mathrm{C}$, choose $t_{0}=0$ and $t_{1}=1$ for the maximum.) This scalar can be seen to measure how far $A$ is from being algebraic of degree two. For the maximum curvature in terms of (11) for $n>2$, take $A$ to be the unitary forward shift. ${ }^{8}$ Then $\left(I-\mathbf{P}_{\mathscr{V}}\right) A^{2}=A^{2}$.

Theorem 3.5 can be stated locally, in terms of the quadratic function (12), yielding probably the simplest way of checking the invertibility of a nonsingular matrix subspace $\mathscr{V}$. Here it is of use to observe that if $\mathscr{V}$ does not contain the scalars, then one can consider the equivalent matrix subspace $\mathscr{V} V^{-1}$ for an invertible $V \in \mathscr{V}$. Clearly, $\mathscr{V} V^{-1}$ contains the scalars. It is also invertible if and only if $\mathscr{V}$ is. ${ }^{9}$

\footnotetext{
${ }^{8}$ The entries on the first subdiagonal and at the position $(1, n)$ are ones.

${ }^{9}$ Besides the linear map $V \mapsto X V Y^{-1}$ with fixed $X, Y \in \mathrm{GL}(n, \mathrm{C})$, it is an interesting problem to characterize maps that preserve invertibility of matrix subspaces.
} 
Corollary 3.8. Assume a matrix subspace $\mathscr{V}$ of $\mathrm{C}^{n \times n}$ over C (or R) contains the scalars. Then $\mathscr{V}$ is invertible if and only if the quadratic function (12) vanishes identically.

Proof. We have already shown that if $\mathscr{V}$ is invertible, then the quadratic function (10) vanishes identically. Hence so does (12).

For the converse, if (12) vanishes identically, then $V^{2} \in \mathscr{V}$ for every $V \in \mathscr{V}$. Inductively, we can conclude that all the even powers of $V$ belong to $\mathscr{V}$, in addition to the first degree polynomials. Consequently,

$$
\left(V+V^{2 j}\right)^{2}=V^{2}+2 V^{2 j+1}+V^{4 j} \in \mathscr{V} \quad \text { for } \quad j=1,2, \ldots
$$

Since the even powers belong to $\mathscr{V}$, it follows that also all the odd powers belong to $\mathscr{V}$ as well. Therefore $\mathscr{V}$ is polynomially closed and hence the matrix subspace $\mathscr{V}$ contains the inverses of its invertible elements.

Example 3.9 ([13]). Let $\mathscr{V} \subset \mathrm{C}^{n \times n}$ be the subspace of Toeplitz matrices (over C) with $n \geq 2$. Recall that $\mathscr{V}$ contains the scalars. Take $V \in \mathscr{V}$ and denote by $t_{j}$ its entries on the $j$ th diagonal, for $-n+1 \leq j \leq n-1$. Then the difference between the $(1,1)$ and $(2,2)$ entries of $V^{2}$ is $t_{n-1} t_{-n+1}-t_{-1} t_{1}$. Hence $V^{2} \notin \mathscr{V}$ generically. Consequently, $\mathscr{V}$ is not invertible.

Lie and Jordan algebras are two of the most important examples of nonassociative (with respect to the product) algebras [14]. Recall that the Jordan product of two matrices $V, W \in \mathrm{C}^{n \times n}$ is defined as

$$
V \bullet W=\frac{1}{2}(V W+W V) .
$$

For a wealth of information on the Jordan product, see [14], [17]. (See also the original paper [15].) A matrix subspace $\mathscr{V}$ over $C$ (or R) is a Jordan subalgebra if the Jordan product is closed in $\mathscr{V}$.

Corollary 3.10. A nonsingular matrix subspace $\mathscr{V}$ of $\mathrm{C}^{n \times n}$ over $\mathrm{C}$ (or $\mathrm{R})$ is invertible if and only if there is a Jordan subalgebra in $O(\mathscr{V})$.

Proof. Suppose $\mathscr{V}$ is invertible. For an invertible element $V \in \mathscr{V}$, consider the matrix subspace $\mathscr{W}=\mathscr{V} V^{-1}$. Then $\mathscr{W} \in O(\mathscr{V})$ and $\mathscr{W}$ contains the scalars. Since $\mathscr{W}$ is invertible as well, for any $V, W \in \mathscr{W}$ the powers $V^{2}$, $W^{2}$ and $(V+W)^{2}$ belong to $\mathscr{W}$. Hence, by computing the difference $(V+$ $W)^{2}-V^{2}-W^{2}$, we can conclude that so does the Jordan product of $V$ and $W$. Consequently, $\mathscr{W}$ is a Jordan subalgebra.

For the converse, suppose $\mathscr{W} \in O(\mathscr{V})$ is a Jordan subalgebra. Take $V=W$ to conclude that $W^{2} \in \mathscr{W}$. By repeating this argument, it follows that all the strictly even powers of $W$ belong to $\mathscr{W}$. By using (13), we can conclude that all 
the odd powers belong to $\mathscr{W}$ as well. Hence we obtain $\operatorname{span}\left\{W, W^{2}, W^{3}, \ldots\right\} \subset$ $\mathscr{W}$. If $W$ is an invertible matrix, then $\operatorname{span}\left\{I, W, W^{2}, \ldots\right\}$ contains the inverse of $W$. Since

$$
\operatorname{span}\left\{W, W^{2}, W^{3}, \ldots\right\}=W \operatorname{span}\left\{I, W, W^{2}, \ldots\right\},
$$

necessarily $I \in \operatorname{span}\left\{W, W^{2}, W^{3}, \ldots\right\}$. Therefore also $\operatorname{span}\left\{W, W^{2}, W^{3}, \ldots\right\}$ and hence $\mathscr{W}$ contains the inverse of $W$. Thus $\mathscr{W}$ is invertible. Consequently, $\mathscr{V}$ is invertible.

COROllary 3.11. A nonsingular matrix subspace $\mathscr{V}$ over $\mathrm{C}($ or $\mathrm{R})$ is a Jordan subalgebra if and only if $\mathscr{V}$ is invertible and contains the scalars.

Consequently, for a nonsingular matrix subspace $\mathscr{V}$ containing the scalars, the function (12) can also be viewed to measure how far $\mathscr{V}$ is from being a Jordan subalgebra. (Finding a nearest Jordan subalgebra to $\mathscr{V}$ is seemingly hard, though.)

COROLlaRY 3.12. Suppose a nonsingular matrix subspace $\mathscr{V}$ over $\mathrm{C}$ (or $\mathrm{R})$ is a Jordan subalgebra. If $V \in \mathscr{V}$ is invertible, then $\mathscr{V} V^{-1}$ (resp. $V^{-1} \mathscr{V}$ ) is a Jordan subalgebra.

As is well known, we have a Lie subalgebra once the sum operation in (14) is replaced with the subtraction, and the resulting product is closed in $\mathscr{V}$. Unlike a Jordan subalgebra with invertible elements, a Lie subalgebra need not be invertible.

EXAMPLE 3.13. The Lie subalgebra $\mathscr{V}_{0} \subset \mathrm{C}^{n \times n}$ of zero trace matrices is nonsingular but not invertible. With $V=\operatorname{diag}(1, \ldots, 1,-(n-1)) \in \mathscr{V}_{0}$, consider $\mathscr{V}_{0} V^{-1}$ containing the scalars. It a simple task to come up with a diagonal element of $\mathscr{V}_{0} V^{-1}$ whose square is not in $\mathscr{V}_{0} V^{-1}$. Using Corollary 3.8 we can conclude that $\mathscr{V}_{0}$ is not invertible.

Aside from nonassociative algebras, also in statistics a related notion of quadratic matrix subspaces of real symmetric matrices has been introduced in [23]. See also [22, Chapter 13]. For such a subspace $\mathscr{V}$ of real symmetric matrices, the function (12) vanishes.

If $\mathscr{V}$ and $\mathscr{W}$ are nonsingular equivalent matrix subspaces with $\mathscr{W}=$ $X \mathscr{V} Y^{-1}$, then the associated bijection

$$
V^{-1} \longmapsto Y V^{-1} X^{-1}
$$

between $\operatorname{Inv}(\mathscr{V})$ and $\operatorname{Inv}(\mathscr{W})$ is smooth. In terms of this map we have the following proposition. 
Proposition 3.14. Assume $\mathscr{V}$ is a nonsingular matrix subspace over $\mathrm{C}$ (or R). If $X$ and $Y$ are unitary, then $\operatorname{Inv}(\mathscr{V})$ and $\operatorname{Inv}\left(X^{\mathscr{V}} Y^{-1}\right)$ have the same curvature at the respective points $V^{-1}$ and $Y V^{-1} X^{-1}$.

Proof. If $X$ and $Y$ are unitary matrices, then the map (15) on $C^{n \times n}$ is also unitary and thereby it preserves the angles between matrices, as well as their norms. Consider the second derivative term $V^{-1}\left(W V^{-1} W-U\right) V^{-1}$ in (7) and the second derivative term $Y V^{-1}\left(W V^{-1} W-U\right) V^{-1} X^{-1}$ of the curve $(V+c(t))^{-1}$ after the map (15) has been applied. By unitarity, the position between them and the respective tangent spaces $V^{-1} \operatorname{Inv}(\mathscr{V}) V^{-1}$ and $Y V^{-1} \operatorname{Inv}(\mathscr{V}) V^{-1} X^{-1}$ is the same.

For instance, it is sufficient to inspect the curvature of either the set of Toeplitz or the set of Hankel matrices considered in Examples 2.7 and 3.9, i.e., the sets of their inverses have the same curvature.

Example 3.15. Let $\mathscr{V}=I \otimes \mathscr{V}$ with a matrix subspace $\mathscr{V} 1$ of $\mathrm{C}^{n_{1} \times n_{1}}$ over $\mathrm{C}($ or R). Then $\mathscr{V}$ is unitarily equivalent to $\mathscr{V} \otimes I[11$, p. 260]. Moreover, by the properties of the Kronecker product concerning inversion, it is clear that that the curvature of $\operatorname{Inv}(\mathscr{V})$ determined by the curvature of $\mathscr{V}$.

Aside from the invertible case, we do not have an understanding of how the curvature behaves in a nonunitary equivalence.

To end this section, let us note that although we have only dealt with matrix subspaces, the concepts introduced make sense also in other applications (as well as in a more abstract setting). In approximation theory, the underlying algebra is typically a function space and hence commutative. For instance, consider the set of analytic functions defined on a domain of $\mathrm{C}$. Then, typically, the finite dimensional subspaces appearing consist of polynomials or rational functions of certain degree at most.

\section{The zero fiber of $\operatorname{Inv}(\mathscr{V})$}

In algebraic geometry, the tangent cone is a classical tool to analyze singularities [10], [6]. For another geometrical tool, the so-called Nash fiber at a singular point of a variety is the set of limits of tangent spaces computed at smooth points; see [18] and references therein.

For an analogy of the Nash fiber, assume $\mathscr{V}$ is a nonsingular matrix subspace of $\mathrm{C}^{n \times n}$ over C (or R). Unlike a variety, $\operatorname{Inv}(\mathscr{V})$ is not closed. Being a smooth manifold, the set of limits of its tangent spaces can yield something of interest only at the boundary points, as is the case for the singular points of a variety. Since $\operatorname{Inv}(\mathscr{V})$ is a homogeneous set, its structure is completely determined in an arbitrarily small neighbourhood of the zero matrix. Therefore the zero 
matrix emerges as a natural boundary point to study in assessing the curvature of $\operatorname{Inv}(\mathscr{V})$ globally. By homogeneity, the set of limits of tangent spaces at the zero matrix is exactly the set of tangent spaces of $\operatorname{Inv}(\mathscr{V})$.

Definition 4.1. Assume $\mathscr{V}$ is a nonsingular matrix subspace over $\mathrm{C}$ (or R). The union of the tangent spaces of $\operatorname{Inv}(\mathscr{V})$ is called the zero fiber of $\operatorname{Inv}(\mathscr{V})$.

Expressed in terms of functions, we are interested in the map

$$
\operatorname{span}\{V\} \longmapsto V^{-1} \mathscr{V} V^{-1}
$$

from $\mathrm{Gr}_{1}(\mathscr{V})$ to $\mathrm{Gr}_{k}\left(\mathrm{C}^{n \times n}\right)$, whenever defined, i.e., when $V \in \mathscr{V}$ is invertible.

The zero fiber of $\operatorname{Inv}(\mathscr{V})$ consists of a single tangent space if and only if $\mathscr{V}$ is invertible. (Equivalently, (16) is a constant function.) Otherwise we have a set of subspaces whose variation can be analyzed in several ways. The maximum angle between these subspaces would certainly be informative but it seems to be computationally out of reach.

For one approach, set

$$
\mathscr{I}(\mathscr{V})=\operatorname{span} \operatorname{Inv}(\mathscr{V})
$$

with the span taken over C (or R). Observe that if $X, Y \in \mathrm{C}^{n \times n}$ are invertible, then

$$
\mathscr{I}\left(X \mathscr{V} Y^{-1}\right)=Y \mathscr{I}(\mathscr{V}) X^{-1},
$$

i.e., $\mathscr{I}\left(X \mathscr{V} Y^{-1}\right)$ and $\mathscr{I}(\mathscr{V})$ are equivalent. The matrix subspace $\mathscr{I}(\mathscr{V})$ can be regarded as a global linearization of $\operatorname{Inv}(\mathscr{V})$ by the fact that it collects the zero fibre as follows.

THEOREM 4.2. For any nonsingular matrix subspace $\mathscr{V}$ over $\mathrm{C}($ or $\mathrm{R})$ there holds

$$
\mathscr{I}(\mathscr{V})=\operatorname{span}_{V^{-1} \in \operatorname{Inv}(\mathscr{V})} V^{-1} \mathscr{V} V^{-1} .
$$

Proof. It is clear that $\mathscr{I}(\mathscr{V}) \subset \operatorname{span}_{V^{-1} \in \operatorname{Inv}(\mathscr{V})} V^{-1 \mathscr{V}} V^{-1}$ since any $V^{-1} \in$ $\operatorname{Inv}(\mathscr{V})$ belongs to $V^{-1 \mathscr{V}} V^{-1}$ by the fact that $V^{-1}=V^{-1} V V^{-1}$.

For the converse inclusion, take $M=\sum_{j=1}^{l} M_{j}$, where $M_{j} \in \mathrm{T}_{V_{j}^{-1}} \operatorname{Inv}(\mathscr{V})$, i.e., $M_{j}=V_{j}^{-1} \hat{V}_{j} V_{j}^{-1}$ for $V_{j}, \hat{V}_{j} \in \operatorname{Inv}(\mathscr{V})$. It suffices to prove that $M_{j} \in$ $\mathscr{I}(\mathscr{V})$. For this, we have

$$
\frac{1}{t}\left(\left(V_{j}-t \hat{V}_{j}\right)^{-1}-V_{j}^{-1}\right)=V_{j}^{-1} \hat{V}_{j} V_{j}^{-1}+O(t)
$$

by using the Neumann series. This proves the claim as $t$ approaches zero by the fact that subspaces of $\mathrm{C}^{n \times n}$ are closed. 
We have $\mathscr{V} \subset \mathscr{I}(\mathscr{V})$ if $\mathscr{V}$ contains the scalars but not necessarily otherwise. Observe that if $\mathscr{V}$ is invertible, then $\mathscr{V}^{-1}=\mathscr{I}(\mathscr{V})$. Therefore, to inspect the curvature of $\operatorname{Inv}(\mathscr{V})$ globally, consider the difference $\operatorname{dim} \mathscr{I}(\mathscr{V})-\operatorname{dim} \mathscr{V}$ to measure how much its tangent spaces vary in all.

Corollary 4.3. There holds $\operatorname{dim} \mathscr{I}(\mathscr{V}) \geq \operatorname{dim} \mathscr{V}$, and

$$
\operatorname{dim} \mathscr{I}(\mathscr{V})=\operatorname{dim} \mathscr{V}
$$

if and only if $\mathscr{V}$ is invertible.

This yields a natural concept for matrix subspaces. Only the case $\operatorname{dim} \mathscr{V} \geq 2$ is of interest by the fact that the equality always holds with $\operatorname{dim} \mathscr{V}=1$.

Definition 4.4. Let $\mathscr{V}$ be a nonsingular matrix subspace of $C^{n \times n}$ over $C$ (or R). Then the grade of $\mathscr{V}$ is $\operatorname{dim} \mathscr{I}(\mathscr{V})$.

Bounding the grade from above is apparently nontrivial. The smallest invertible matrix subspace containing $\mathscr{V}$ is fit for this purpose. Also, the subalgebra generated by $\mathscr{V}$ obviously contains $\mathscr{I}(\mathscr{V})$. The use of this subalgebra does not seem to be natural since the construction misses the respective equivalence relation (6) which we regard as fundamental. ${ }^{10}$ In all, these sets are not readily generated and, even then, they may provide very pessimistic bounds.

ExAmple 4.5. Let $\mathscr{V}$ be the matrix subspace of $\mathrm{C}^{n \times n}$ over $\mathrm{C}$ spanned by $V_{1}$, the forward unitary shift, ${ }^{11}$ and $V_{2}=\operatorname{diag}\left(1, \omega, \ldots, \omega^{n-1}\right)$ with $\omega=e^{2 \pi i / n}$. The subalgebra generated by the matrices $V_{1}$ and $V_{2}$ is $C^{n \times n}$.

Consequently, the subalgebra generated already by a two dimensional matrix subspace $\mathscr{V}$ can be of dimension $n^{2}$. Next we show that this is in sharp contrast with the actual grade of $\mathscr{V}$. For this, denote by $\operatorname{deg}(V)$ the degree of the minimal polynomial of $V \in \mathscr{V}$. (The degree depends on whether $\mathscr{V}$ is regarded as a matrix subspace over $\mathrm{C}$ or R.)

THeOREM 4.6. Let $\mathscr{V}$ be a nonsingular matrix subspace of $\mathrm{C}^{n \times n}$ over $\mathrm{C}$ (or $\mathrm{R})$ with $\operatorname{dim} \mathscr{V}=2$. Then the grade of $\mathscr{V}$ equals $\operatorname{deg}\left(V_{2} V_{1}^{-1}\right)$ for any $V_{1} \in \mathscr{V} \cap \mathrm{GL}(n, \mathrm{C})$ and $V_{2} \in \mathscr{V}$ spanning $\mathscr{V}$.

PRoof. With $X=I$ and $Y=V_{1}$ consider $\mathscr{W}=X \mathscr{V} Y^{-1}=\operatorname{span}\{I, W\}$, where $W=V_{2} V_{1}^{-1}$. Recall that $\operatorname{Inv}\left(X \mathscr{V} Y^{-1}\right)=Y \operatorname{Inv}(\mathscr{V}) X^{-1}$ for any invertible matrices $X, Y \in \mathrm{C}^{n \times n}$. Hence, since $\operatorname{dim} \mathscr{I}(\mathscr{V})$ is invariant for equivalent matrix subspaces, we can consider the grade of $\mathscr{W}$. Clearly, this a matrix subspace with commuting elements.

\footnotetext{
${ }^{10}$ In [1] subalgebras are used to classify finite dimensional operator systems.

${ }^{11}$ The forward unitary shift is the permutation matrix having ones on its first subdiagonal and at the position $(1, n)$.
} 
Observe first that, whenever invertible, the inverse of $\lambda I-W$ is a polynomial in $\lambda I-W$ and hence a polynomial in $W$. Therefore $\mathscr{I}(\mathscr{W})$ consists of polynomials in $W$. It remains to show that all the polynomials in $W$ are in $\mathscr{I}(\mathscr{W})$.

Clearly, any power of $(\lambda I-W)^{-1}$ is also a polynomial in $W$. Differentiating the resolvent operator $(\lambda I-W)^{-1}$ gives

$$
\frac{d^{j}}{d \lambda^{j}}(\lambda I-W)^{-1}=(-1)^{j} j !(\lambda I-W)^{-(j+1)} .
$$

Being limits of difference quotients, these derivatives belong to $\operatorname{Inv}(\mathscr{W})$. By the fact that $W$ and $(\lambda I-W)^{-1}$ have the same degree, $\mathscr{I}(\mathscr{W})$ consists of all the polynomials in $W$. Thereby the dimension of $\mathscr{I}(\mathscr{W})$ equals the degree of $W$.

Example 4.7. Consider again Example 2.4. The generalized eigenvalue problem (3) is concerned with the singular elements of the associated two dimensional matrix subspace $\mathscr{V}$. (The singular elements determine the spectrum of a matrix subspace [13, Section 3].) Usually the problem is converted into an equivalent matrix subspace $\mathscr{W}=\operatorname{span}\{I, W\}$ with commuting elements. Being equal to the grade of $\mathscr{V}$, the degree of the matrix $W$ is independent of the matrix $Y \in \mathscr{V}$ used in the equivalence.

By Proposition 2.5, the case $\mathrm{Gr}_{2}\left(\mathrm{C}^{n \times n}\right)$ is very exceptional by being easier to analyze. Benefiting from commutativity, it can be dealt with, to large extent, by using classical tools of matrix analysis for a single matrix. General results for $k>2$ are not readily given.

Observe that if $\mathscr{V}$ is equivalent to a nonsingular matrix subspace $\mathscr{W}$ with commuting elements, then the dimension of $\mathscr{V}$ cannot be arbitrary. (For showing the existence of such a $\mathscr{W}$, see the proof of Proposition 2.5, which is constructive.) It is bounded from above by the dimension of the nullspace of the linear operator

$$
M \longmapsto W M-M W
$$

on $\mathrm{C}^{n \times n}$, for any fixed $W \in \mathscr{W}$. The obvious reason is that the nullspace contains $\mathscr{W}$. For the dimension of the nullspace of (18), see [11, p. 275]. In particular, if there is a nonderogatory element $W$ in $\mathscr{W}$, then all the elements in the nullspace of (18) are polynomials in $W$ and the grade of $\mathscr{V}$ equals the degree of $W$. (For this, the proof of Theorem 4.6 applies.) With this, one can readily construct matrix subspaces $\mathscr{V}$ satisfying

$$
\operatorname{dim} \mathscr{I}(\mathscr{V})-\operatorname{dim} \mathscr{V}=n-j
$$

with $\operatorname{dim} \mathscr{V}=j \geq 2$. 


\section{Conclusions}

Square matrix subspaces are either singular or nonsingular, with the latter property being generic. Nonsingular matrix subspaces can, in turn, be separated into being invertible or into those whose set of inverses has a nonvanishing curvature. For the nonvanishing case, there are several alternatives to measure curvature. The second fundamental form of Riemannian geometry was given and used to link invertible matrix subspaces with Jordan subalgebras. Global measures were also suggested. Regarding $\operatorname{Gr}_{k}\left(C^{n \times n}\right)$, the case $k=2$ is exceptional. The noncommutativity is fundamentally present only for $k>2$.

ACKNOWLEDGEMENTS. I would like to thank the referee for his/her remarks.

\section{REFERENCES}

1. Arveson, W., The noncommutative Choquet boundary III: Operator systems in matrix algebras, Math. Scand. 106 (2010), 196-210.

2. Asplund, E., Inverses of matrices $a_{i j}$ which satisfy $a_{i j}=0$ for $j>i+p$, Math. Scand. 7 (1959), 57-60.

3. Asplund, S. O., Finite boundary value problems solved by Green's matrix, Math. Scand. 7 (1959), 49-56.

4. Byckling, M., and Huhtanen, M., Approximate factoring of the inverse, to appear in $\mathrm{Nu}-$ mer. Math.

5. Causin, A., and Pirola, G. P., A note on spaces of symmetric matrices, Linear Algebra Appl. 426 (2007), 533-539.

6. Cox, D., Little, J., and O'Shea, D., Ideals, Varieties and Algorithms. An Introduction to Computational Algebraic Geometry and Commutative Algebra, 2nd ed., Springer, New York 1997.

7. Edelman, A., Arias, T., and Smith, S., The geometry of algorithms with orthogonality constraints, SIAM J. Matrix Anal. Appl. 20 (1999), 303-353.

8. Effros, E. G., and Ruan Z.-J., Operator Spaces, London Math. Soc. Monographs (N.S.) 23, Oxford Univ. Press, Oxford 2000.

9. Gantmacher, F.R., The Theory of Matrices II, Chelsea, New York 1959.

10. Harris, J., Algebraic Geometry. A First Course, Graduate Texts in Math. 133, Springer, New York 1992.

11. Horn, R. A., and Johnson, C. R., Topics in Matrix Analysis, Cambridge Univ. Press, Cambridge 1991.

12. Huhtanen, M., Factoring matrices into the product of two matrices, BIT 47 (2007), 793-808.

13. Huhtanen, M., Matrix subspaces and determinantal hypersurfaces, Ark. Mat. 48 (2010), $57-77$.

14. Jacobson, N., Basic Algebra I, Freeman, San Francisco 1974.

15. Jordan, P., Über Verallgemeinerungsmöglichkeiten des Formalismus der Quantenmechanik, Nachr. Akad. Wiss. Göttingen. Math. Phys. Kl. I 41 (1933), 209-217.

16. Lee, J. M., Riemannian Manifolds. An Introduction to Curvature, Graduate Texts in Math. 176, Springer, New York 1997.

17. McCrimmon, K., A Taste of Jordan Algebras, Universitext, Springer, New York 2004. 
18. O'Shea, D., and Wilson, L., Limits of tangent spaces to real surfaces, Amer. J. Math. 126 (2004), 951-980.

19. Parlett, B., The Symmetric Eigenvalue Problem, Classics in Applied Math. 20, SIAM, Philadelphia 1997.

20. Paulsen, V., Completely Bounded Maps and Operator Algebras, Cambridge Studies in Advanced Math. 78, Cambridge Univ. Press, Cambridge 2002.

21. Piontkowski, J., Linear symmetric determinantal hypersurfaces, Michigan Math. J. 54 (2006), 117-146.

22. Rao, C. R., and Rao, M. B., Matrix Algebra and Its Applications to Statistics and Econometrics, World Scientific, River Edge, NJ 1998.

23. Seely, J., Quadratic subspaces and completeness, Ann. Math. Statist. 42 (1971), 710-721.

24. Vandebril, R., Van Barel, M., Golub, G., and Mastronardi, N., A bibliography on semiseparable matrices, Calcolo 42 (2005), 249-270.

INSTITUTE OF MATHEMATICS

HELSINKI UNIVERSITY OF TECHNOLOGY

BOX 1100

FIN-02015

FINLAND

E-mail: Marko.Huhtanen@tkk.fi 